\title{
Mandenkan
}

MANDENIKAN Bulletin semestriel d'études linguistiques mandé

49 | 2013

Le maninka du Niokolo

\section{Catégories lexicales majeures}

Section 5

Denis Creissels

\section{OpenEdition}

Journals

Édition électronique

URL : https://journals.openedition.org/mandenkan/601

DOI : 10.4000/mandenkan.601

ISSN : 2104-371X

Éditeur

Llacan UMR 8135 CNRS/Inalco

Édition imprimée

Date de publication : 1 juin 2013

Pagination : 44-50

ISSN : 0752-5443

Référence électronique

Denis Creissels, «Catégories lexicales majeures », Mandenkan [En ligne], 49 | 2013, mis en ligne le 25 avril 2014, consulté le 16 janvier 2023. URL : http://journals.openedition.org/mandenkan/601 ; DOI : https://doi.org/10.4000/mandenkan.601

Ce document a été généré automatiquement le 16 janvier 2023.

\section{(c) $)(1)(2)$}

Creative Commons - Attribution - Pas d'Utilisation Commerciale - Partage dans les Mêmes Conditions 4.0 International - CC BY-NC-SA 4.0

https://creativecommons.org/licenses/by-nc-sa/4.0/ 


\title{
Catégories lexicales majeures
}

\author{
Section 5
}

Denis Creissels

\subsection{Noms et verbes, et la nominalisation de procès}

1 Pour ce qui est de la distinction entre noms et verbes, la situation en maninka du Niokolo n'est pas différente de celle observée en mandinka, et on pourrait ici reprendre point par point ce qui est dit pour le mandinka dans Creissels \& Sambou (2013). En résumé, la règle générale est que les lexèmes verbaux peuvent sans aucun marquage morphologique particulier s'employer comme noms de procès. Dans cet emploi, où comme n'importe quel nom ils ont la forme déterminée comme forme par défaut, ils se combinent notamment avec des dépendants génitivaux représentant leurs arguments nucléaires. Comme les autres variétés mandingues, le maninka du Niokolo a deux variantes de la construction génitivale (cf. 14), et avec les lexèmes verbaux utilisés comme noms de procès, la variante par juxtaposition directe est utilisée si le dépendant génitival transpose l'objet d'une construction transitive - ex. (30), tandis que la construction faisant intervenir la postposition la est utilisée si le dépendant génitival transpose un sujet - ex. (31).

\begin{tabular}{|l|l|l|l|l|l|l|}
\hline (30) & a. & Kóntín-o & móy-ǒo & ye & dii & lée. \\
\hline & & Konting-D & entendre-D & ACPP & être_agréable & FOC \\
\hline & & 'Le konting (sorte de guitare) est agréable à entendre.' \\
\hline & b. & Síy-ǒo & lu & jěe & măy & di-yaa. \\
\hline & & buffle-D & PL & voir.D & ACPN & être_facile-ABSTR \\
\hline & & 'Ce n'est pas facile de voir des buffles. \\
\hline
\end{tabular}




\begin{tabular}{|c|c|c|c|c|c|c|c|c|c|c|}
\hline (31) & a. & $\eta$ & \multicolumn{3}{|l|}{ peletí-ta } & á & la & \multicolumn{2}{|c|}{ ina } & la. \\
\hline & & $1 \mathrm{SG}$ & \multicolumn{2}{|c|}{ être_sûr-ACPP } & & $3 S G$ & & \multicolumn{2}{|c|}{ venir.D } & OBL \\
\hline & & \multicolumn{9}{|c|}{ 'Je suis sûr de sa venue.' } \\
\hline & b. & \multicolumn{2}{|l|}{ Tabi-ri-láa } & lée & folo & se & kin-óo & la & di-yáa & log. \\
\hline & & \multicolumn{2}{|c|}{ cuire-ANTIP-NMAG.D } & FOC & premier.D & РОТР & plat-D & GEN & être_agréable-ABSTR.D & savoir \\
\hline & & \multicolumn{9}{|c|}{ 'La cuisinière est la première à savoir si le plat est bon.' } \\
\hline
\end{tabular}

2 Comme en mandinka, l'emploi des lexèmes verbaux transitifs comme noms de procès est seulement soumis en règle générale à des contraintes strictes sur l'expression des arguments nucléaires : en l'absence d'expression de l'argument objet, un lexème verbal transitif utilisé nominalement a une interprétation passive, et l'argument sujet ne peut pas être exprimé, sauf si le lexème verbal est élargi d'un suffixe -ri.

\begin{tabular}{|c|c|c|c|c|c|}
\hline (32) & a. & túyu & & & 'piler' \\
\hline & b. & túz-ǒo & & & 'le fait d'être pilé' \\
\hline & & piler-D & & & \\
\hline & c. & ñóo-tuð-óo & & & 'le fait de piler du mil' \\
\hline & & mil-piler-D & & & \\
\hline & d. & mús-ǒo & la & ñóo-tuð-óo & 'le fait que la femme pile du mil' \\
\hline & & femme-D & GEN & mil-piler-D & \\
\hline & e. & *mús-ǒo la túy-ǒo & & & \\
\hline & f. & túzu-r-óo & & & 'le fait de piler' \\
\hline & & piler-ANTIP-D & & & \\
\hline & g. & mús-ǒo & la & 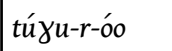 & 'le fait que la femme pile' \\
\hline & & femme-D & GEN & piler-ANTIP-D & \\
\hline
\end{tabular}

3 Le suffixe -ri n'est jamais nécessaire pour qu'un lexème verbal puisse être utilisé nominalement, et il ne se combine qu'à des lexèmes verbaux transitifs. Il n'a donc pas pour fonction de marquer la nominalisation, mais uniquement de réguler l'expression de la structure argumentale des verbes transitifs, et il le fait d'une façon qui permet de l'identifier comme un opérateur d'antipassif - cf. Creissels \& Sambou (2013) pour une discussion plus détaillée. 
4 Parmi les verbes transitifs, se' n'ayant pas de forme antipassive et s'employant à la forme non-dérivée dans des conditions où les verbes transitifs prennent normalement la forme antipassive.

\subsection{Verbes qualificatifs}

\subsubsection{La notion de verbe qualificatif}

5 En ce qui concerne les contrastes entre catégories lexicales majeures, le seul point qui mérite d'être développé dans une esquisse du maninka du Niokolo concerne la classe des verbes qualificatifs, qui n'a pas d'équivalent en mandinka, et qui ne se présente pas non plus exactement de la même façon que dans les autres variétés de mandingue pour lesquelles des descriptions sont disponibles.

6 L'étiquette 'verbe qualificatif', qui se justifie par le sémantisme de la plupart des verbes qui se rattachent à cette classe, s'applique ici aux lexèmes qui expriment une valeur d'état en se combinant au couple de prédicatifs ye / may utilisé par ailleurs pour marquer l'accompli-statif en construction transitive, et non pas au couple - 'ta / may qui marque en règle générale l'accompli-statif dans la construction intransitive. Certains des lexèmes qui ont cette aptitude n'ont pas d'autre possibilité de fonctionnement prédicatif, et n'ont pas non plus les formes non finies décrites en 7 , alors que d'autres ont par ailleurs sans aucune restriction le fonctionnement de verbes ordinaires.

7 Comme les exemples cités en (33) le montrent, le statut de verbe qualificatif concerne en maninka du Niokolo non seulement les lexèmes qui se rencontrent couramment avec ce statut dans les autres variétés mandingues, mais aussi un certain nombre de lexèmes qui n'ont généralement pas ce statut.

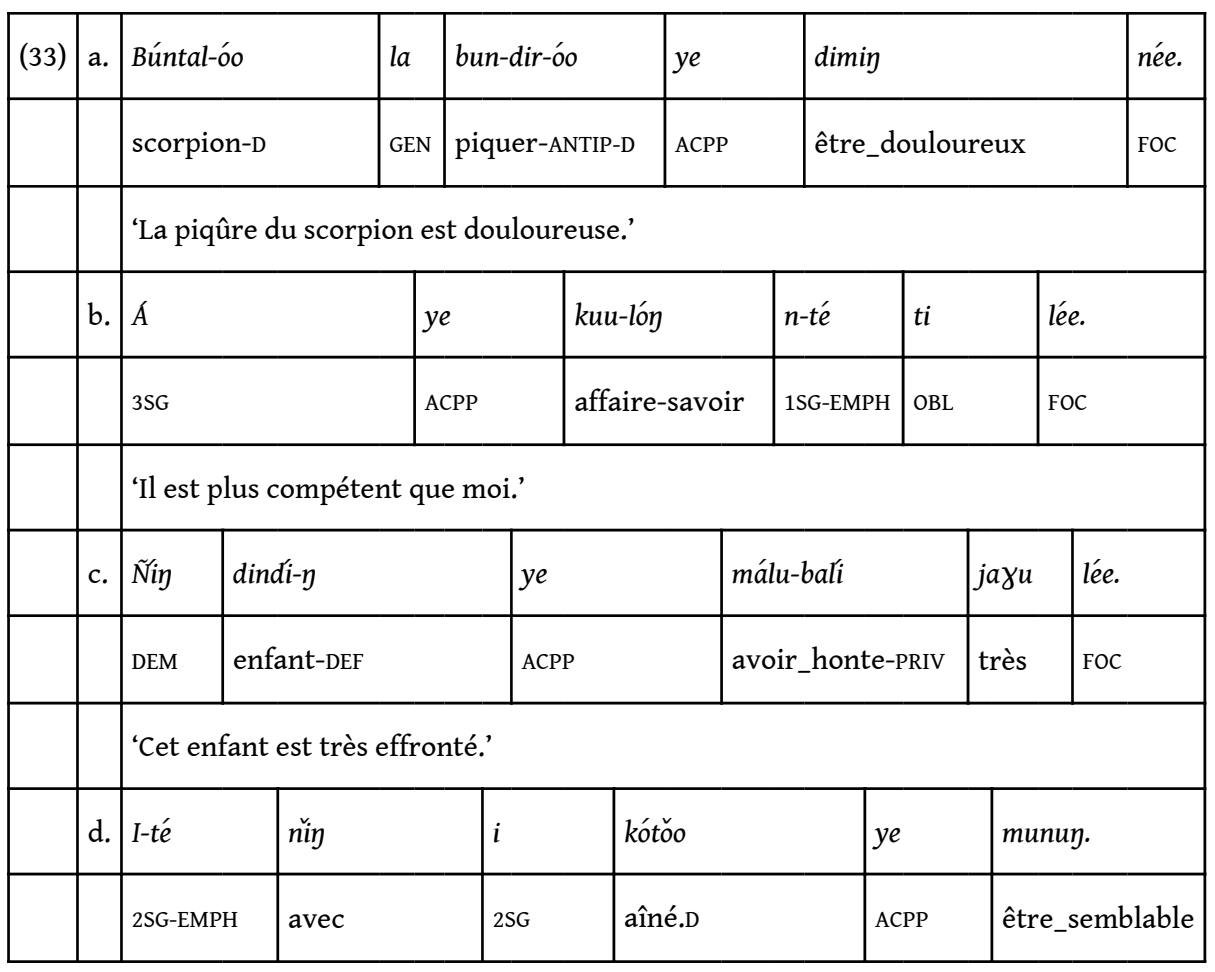


Par rapport à d'autres variétés mandingues où une telle catégorie existe, on notera aussi qu'elle ne se manifeste pas par des marqueurs prédicatifs particuliers, mais seulement par une règle particulière de sélection de l'une des deux variantes du prédicatif d'accompli-statif positif.

9 Avec les lexèmes qui peuvent fonctionner à la fois comme verbe qualificatif et comme verbe ordinaire, il peut y avoir synonymie entre l'emploi intransitif du prédicatif ye (typique des verbes qualificatifs) et l'emploi de - 'ta (typique des verbes intransitifs ordinaires), comme en (34) et (35).

\begin{tabular}{|l|l|l|l|l|l|}
\hline (34) & a. & Ñoyolo & dánnáa & siyáa-ta. & \\
\hline & & Niokolo & chasseur.D & être_abondant-ACPP & \\
\hline & & \multicolumn{1}{|l|}{ 'Au Niokolo les chasseurs sont nombreux.' } & \\
\hline & b. & Noyolo & dánnáa & ye & siyaa. \\
\hline & & Niokolo & chasseur.D & ACPP & être_abondant \\
\hline & id. & & & \\
\hline
\end{tabular}

\begin{tabular}{|l|l|l|l|l|l|l|}
\hline (35) & a & \multicolumn{1}{|l|}{ Sámǎa } & \multicolumn{2}{|l|}{ wára-ta } & lée. & \\
\hline & & éléphant.D & \multicolumn{2}{|l|}{ être_gros-ACPP } & FOC & \\
\hline & & 'L'éléphant est gros.' & & & & \\
\hline & b. & Sámáa & ye & wára & lée. \\
\hline & & éléphant.D & ACPP & être_gros & FOC \\
\hline & id. & & & \\
\hline
\end{tabular}

10 L'équivalence n'est toutefois pas totale, car des formes comme siyáata ou wárata se rencontrent aussi dans des contextes où elles signifient le résultat d'un processus ('sont devenus nombreux', 'a grandi'), alors que ye siyaa ou ye wára ne peuvent signifier qu'une pure valeur d'état.

Pour les verbes qualificatifs qui ne peuvent se combiner qu'au couple de prédicatifs ye / may, on peut en règle générale dériver un verbe ordinaire par suffixation de -yaa, avec la même relation sémantique : le verbe obtenu par suffixation de -yaa peut lui aussi s'utiliser à l'accompli-statif avec une valeur stative, comme diyaa à l'ex. (36), mais en fonction du contexte, la forme diyáata est apte aussi à rendre 'être devenu bon', alors que ye dii ne peut avoir qu'une valeur purement stative. 


\begin{tabular}{|l|l|l|l|l|l|}
\hline (36) & a. & Bála-sub-óo & ye & dii. & \\
\hline & & porc_épic-viande-D & ACPP & être_agréable & \\
\hline & & 'La viande de porc-épic est bonne.' & \\
\hline & b. & Bála-sub-óo & di-yáa-ta. \\
\hline & & porc_épic-viande-D & être_agréable-ABSTR-ACPP \\
\hline & & id. & \\
\hline
\end{tabular}

\subsubsection{L'inventaire des verbes qualificatifs}

12 Les lexèmes suivants ont été relevés avec le comportement de verbes qualificatifs :

\begin{tabular}{|l|l|}
\hline basi & 'être aigre, acide' \\
\hline bete & 'être bon' \\
\hline buy & 'être gros' \\
\hline dii & 'être agréable, facile' \\
\hline dimin & 'être douloureux' \\
\hline doyo & 'être petit' \\
\hline fáni & 'être menteur' \\
\hline fása & 'être dur' \\
\hline fati & 'être audacieux' \\
\hline fee & 'être léger' \\
\hline fisa & 'être meilleur' \\
\hline fúya & 'être mou' \\
\hline fúuru & 'être paresseux' \\
\hline jayu & 'être mauvais, méchant' \\
\hline jiitoo & 'être peureux' \\
\hline
\end{tabular}




\begin{tabular}{|c|c|}
\hline káayee & 'être peureux' \\
\hline kay & 'être égal' \\
\hline kéebaa & 'être âgé’ \\
\hline kéejazu & 'être laid' \\
\hline kéeñin & ‘être joli \\
\hline kende & 'être en bon état, en bonne santé' \\
\hline kéwu & 'être malin’ \\
\hline kóle & 'être difficile' \\
\hline ko to & 'être vieux' \\
\hline kuli & 'être lourd' \\
\hline kuna & 'être amer’ \\
\hline kuruy & 'être mauvais' \\
\hline kuu & ‘être désagréable’ \\
\hline kuulandii & 'être un faiseur d'histoires' ${ }^{1}$ \\
\hline kuuloy & 'savoir des choses, être informé' \\
\hline lázasa & 'être mauvais' \\
\hline máadii & 'être aimé, sympathique’2 \\
\hline máakuu & 'être antipathique' \\
\hline málubali & 'être effronté' \\
\hline mée & 'être avide de viande' \\
\hline mese & 'être mince, fin' \\
\hline munuy & 'être semblable' \\
\hline nama & 'être gluant, glissant' \\
\hline$\tilde{n} i \eta$ & 'être beau, bien' \\
\hline sála & 'être paresseux' \\
\hline see & 'être fade' \\
\hline senu & 'être propre' \\
\hline
\end{tabular}




\begin{tabular}{|l|l|l|}
\hline siyaa & 'être abondant' \\
\hline sumaa & 'être froid, frais' \\
\hline sútu & 'être court, proche' \\
\hline tari & 'être rapide, fréquent' \\
\hline timi & 'être doux, sucré' \\
\hline tóolee & 'être stupide' \\
\hline wára & 'être grand' \\
\hline
\end{tabular}

13 Parmi les lexèmes de cette liste qui ont d'autres comportements syntaxiques possibles, tous ne sont pas attestés de façon également productive comme verbes qualificatifs. Dans de tels cas, on observe généralement que le fonctionnement de verbe qualificatif est particulièrement usuel dans les phrases exprimant une comparaison, comme cela est illustré en (37) par ke -'ebaa, qui fonctionne par ailleurs comme nom ('homme âgé) et comme qualifiant ('âgé').

\begin{tabular}{|l|l|l|l|l|l|l|l|l|l|}
\hline (37) & Júmay & née & ye & kéebaa & ñin & móro & fúláa & lu & to ? \\
\hline & lequel & FOC & ACPP & être_âgé & DEM & personne & deux.D & PL & LOC \\
\hline
\end{tabular}

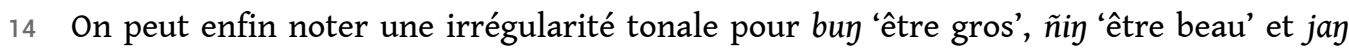
'être long' : en dérivation, ces trois termes se comportent invariablement comme s'ils comportaient un ton haut lexical (cf. búnyaa 'devenir gros', ñiiñaa 'devenir beau', jáyyaa 'devenir long'), alors qu'en tant que verbes qualificatifs, ils ne manifestent aucun ton haut.

\subsection{Les adjectifs}

15 Comme de manière générale en mandingue, il est possible de reconnaître comme adjectifs des lexèmes qui n'ont pas d'emploi prédicatif possible à leur forme non dérivée, qui s'emploient typiquement comme qualifiants de noms, et qui peuvent aussi s'employer nominalement, mais seulement dans des contextes où on peut les interpréter comme qualifiant un nom suggéré par le contexte. C'est par exemple le cas de kuta 'neuf, nouveau'. 


\subsection{Contraintes sur la structure syllabique des lexèmes}

16 Comme en mandinka, la règle générale est que les lexèmes verbaux, nominaux et adjectivaux monosyllabiques ne peuvent pas être constitués d'une syllabe légère, et cette règle admet les mêmes exceptions qu'en mandinka, à savoir six lexèmes verbaux qui selon les contextes ont un allomorphe CV et un allomorphe CVV :

\begin{tabular}{|l|l|l|}
\hline & $b o(o)$ & 'sortir' \\
\hline & $f o(o)$ & 'dire' \\
\hline & $j e(e)$ & 'voir' \\
\hline & $k e(e)$ & 'faire, mettre, devenir' \\
\hline & $s o(o)$ & 'donner' \\
\hline$t u(u)$ & 'laisser' \\
\hline
\end{tabular}

17 L'allomorphe irrégulier CV est sélectionné dans les contextes suivants :

- en fonction de prédicat verbal, en combinaison avec l'un des prédicatifs dont l'inventaire a été donné en 4.2 , ainsi qu'à l'impératif ;

- à l'infinitif en 'ke 'ka;

- à l'infinitif en -'la ;

- à l'infinitif nu employé comme complément de taa 'aller' ou náa 'venir'.

Dans tous les autres contextes, c'est l'allomorphe régulier CVV qui est utilisé. C'est lui qui apparaît au résultatif, au progressif, au gérondif, à l'antipassif, lorsque le lexème verbal est utilisé nominalement, ainsi que dans la formation de lexèmes composés ou dérivés.

\section{NOTES}

1. Avec kuulandii, on a une possibilité de synonymie avec une construction dans laquelle un lexème kuula' qui n'existe pas de manière productive en dehors de cette construction est le sujet de dii 'être agréable' : Á ye kuulandii = Á kuulá ye dii 'Il fait des histoires'.

2. Avec máadii et máakuu, on a une possibilité de synonymie avec une construction dans laquelle un lexème máa qui n'existe pas de manière productive en dehors de cette construction est le sujet de dii 'être agréable' ou kuu 'être désagréable' : Á ye máadii = A máa ye dii 'Il est sympathique', Á ye máakuu = A máa ye kuu 'Il est antipathique'. 


\section{AUTEUR}

DENIS CREISSELS

Université de Lyon

Denis.Creissels@univ-lyon2.fr 